\title{
Relativistic Hartree-Bogoliubov theory with finite range pairing forces in coordinate space: Neutron halo in light nuclei
}

\author{
W. Pöschl, D. Vretenar, G.A. Lalazissis and P. Ring \\ Physics Department, Technische Universität München, D-85748 Garching
}

July 3, 2021

\begin{abstract}
The Relativistic Hartree Bogoliubov (RHB) model is applied in the self-consistent mean-field approximation to the description of the neutron halo in the mass region above the s-d shell. Pairing correlations and the coupling to particle continuum states are described by finite range twobody forces. Finite element methods are used in the coordinate space discretization of the coupled system of Dirac-Hartree-Bogoliubov integrodifferential eigenvalue equations, and Klein-Gordon equations for the meson fields. Calculations are performed for the isotopic chains of $\mathrm{Ne}$ and $\mathrm{C}$ nuclei. We find evidence for the occurrence of neutron halo in heavier $\mathrm{Ne}$ isotopes. The properties of the 1f- $2 \mathrm{p}$ orbitals near the Fermi level and the neutron pairing interaction play a crucial role in the formation of the halo. Our calculations display no evidence for the neutron halo phenomenon in C isotopes.
\end{abstract}

The structure of exotic nuclei with extreme isospin values displays many interesting phenomena: extremely weak binding of the outermost nucleons, coupling between bound states and the particle continuum, regions of neutron halos, large spatial dimensions and the existence of the neutron skin. Modifications of shell structure in dripline nuclei have been predicted, as well as changes in the evolution of collectivity. For dripline nuclei the separation energy of the last nucleons can become extremely small. The Fermi level is found close to the particle continuum, and the lowest particle-hole or particle-particle modes are often embedded in the continuum. The neutron density distribution in such loosely bound nuclei shows an extremely long tail, the neutron halo. The resulting large interaction 
cross sections have provided the first experimental evidence for halo nuclei [1]. The neutron halo phenomenon has been studied with a variety of theoretical models [2, 3]. For very light nuclei in particular, models based on the separation into core plus valence space nucleons (three-body Borromean systems) have been employed. In heavier neutron-rich nuclei one expects that mean-field models should provide a better description of ground-state properties. In a mean-field description, the neutron halo and the stability against nucleon emission can only be explained with the inclusion of pairing correlations. Both the properties of single-particle states near the neutron Fermi level and the pairing interaction are important in the formation of the neutron halo.

Relativistic mean-field models have been successfully applied in calculations of nuclear matter and properties of finite nuclei throughout the periodic table. The theory provides a framework for describing the nuclear many-body problem as a relativistic system of baryons and mesons.. In the self-consistent mean-field approximation, detailed calculations have been performed for a variety of nuclear structure phenomena [4]. The Relativistic Hartree-Bogoliubov (RHB) theory in coordinate space, which is an extension of non-relativistic HFB-theory [5], provides a unified description of mean-field and pairing correlations. The RHB theory has recently been applied in the description of ground-state properties of $\mathrm{Sn}$ and $\mathrm{Pb}$ isotopes [6], using an expansion in a large oscillator basis for the solution of the Dirac-Hartree-Bogoliubov equations. In many applications an expansion of the wave functions in an appropriate oscillator basis of spherical or axial symmetry provides a satisfactory level of accuracy. For dripline nuclei, the expansion in the localized oscillator basis presents only a poor approximation to the continuum states. Oscillator expansions display a slow convergence in the asymptotic region of the coordinate space. In order to correctly describe the coupling between bound and continuum states, the Dirac-Hartree-Bogoliubov equations have to be solved in coordinate space. Recently, a fully self-consistent RHB model in coordinate space has been used to describe the two-neutron halo in ${ }^{11} \mathrm{Li}$ [7]. However, only a density dependent force of zero range has been used in the pairing channel. Finite range forces, as for example the Gogny interaction, should provide a more realistic description of pairing correlations. A very efficient procedure for the coordinate space discretization of the RHB model is provided by Finite Element Methods (FEM). In Refs. [8, 9] we have developed a FEM based model and computer code for the solution of Dirac-Hartree-Bogoliubov equations in coordinate space. In the present work we present the first application of the model in the description of ground state properties of $\mathrm{Ne}$ and $\mathrm{C}$ isotopes. For these elements, isotopes near the neutron drip should soon become accessible in experiments with radioactive beams. Therefore it is extremely important to investigate for which isotopes the relativistic Hartree-Bogoliubov model predicts the formation of neutron halo.

The model describes the nucleus as a system of Dirac nucleons which interact in a relativistic covariant manner through the exchange of virtual mesons [10]: the 
isoscalar scalar $\sigma$-meson, the isoscalar vector $\omega$-meson and the isovector vector $\rho$-meson. The photon field $(A)$ accounts for the electromagnetic interaction. The relativistic extension of the HFB theory is described in Ref. [11]. Independent quasi-particles are introduced and the ground state of a nucleus $|\Phi\rangle$ is represented as the vacuum with respect to these quasi-particles. The quasi-particle operators are defined by a unitary Bogoliubov transformation of the single-nucleon creation and annihilation operators. The generalized single-particle hamiltonian of HFB theory contains two average potentials: the self-consistent field $\hat{\Gamma}$ which encloses all the long range $p h$ correlations, and a pairing field $\hat{\Delta}$ which sums up the $p p$-correlations. In the Hartree approximation for the self-consistent mean field, the Relativistic Hartree-Bogoliubov (RHB) equations read

$$
\left(\begin{array}{cc}
\hat{h}_{D}-m-\lambda & \hat{\Delta} \\
-\hat{\Delta}^{*} & -\hat{h}_{D}+m+\lambda
\end{array}\right)\left(\begin{array}{c}
U_{k} \\
V_{k}
\end{array}\right)=E_{k}\left(\begin{array}{c}
U_{k} \\
V_{k}
\end{array}\right) .
$$

where $\hat{h}_{D}$ is the single-nucleon Dirac hamiltonian, and $m$ is the nucleon mass. $U_{k}$ and $V_{k}$ are quasi-particle Dirac spinors, and $E_{k}$ denote the quasi-particle energies.

The RHB equations are non-linear integro-differential equations. They have to be solved self-consistently, with potentials determined in the mean-field approximation from solutions of Klein-Gordon equations for mesons and Coulomb field:

$$
\begin{aligned}
{\left[-\Delta+m_{\sigma}^{2}\right] \sigma(\mathbf{r})=} & -g_{\sigma} \sum_{E_{k}>0} V_{k}^{\dagger}(\mathbf{r}) \gamma^{0} V_{k}(\mathbf{r}) \\
& -g_{2} \sigma^{2}(\mathbf{r})-g_{3} \sigma^{3}(\mathbf{r}), \\
{\left[-\Delta+m_{\omega}^{2}\right] \omega^{0}(\mathbf{r})=} & g_{\omega} \sum_{E_{k}>0} V_{k}^{\dagger}(\mathbf{r}) V_{k}(\mathbf{r}), \\
{\left[-\Delta+m_{\rho}^{2}\right] \rho^{0}(\mathbf{r})=} & g_{\rho} \sum_{E_{k}>0} V_{k}^{\dagger}(\mathbf{r}) \tau_{3} V_{k}(\mathbf{r}), \\
-\Delta A^{0}(\mathbf{r})= & e \sum_{E_{k}>0} V_{k}^{\dagger}(\mathbf{r}) \frac{1-\tau_{3}}{2} V_{k}(\mathbf{r})
\end{aligned}
$$

The source terms are sums of bilinear products of baryon amplitudes. The sums run over all positive energy states. The system of equations is solved selfconsistently in coordinate space by discretization on the finite element mesh. In the coordinate space representation of the pairing field $\hat{\Delta}$, the kernel of the integral operator is

$$
\Delta_{a b}\left(\mathbf{r}, \mathbf{r}^{\prime}\right)=\frac{1}{2} \sum_{c, d} V_{a b c d}\left(\mathbf{r}, \mathbf{r}^{\prime}\right) \kappa_{c d}\left(\mathbf{r}, \mathbf{r}^{\prime}\right)
$$

where $V_{a b c d}\left(\mathbf{r}, \mathbf{r}^{\prime}\right)$ are matrix elements of a general two-body pairing interaction and $\kappa_{c d}\left(\mathbf{r}, \mathbf{r}^{\prime}\right)$, is the pairing tensor, defined as

$$
\kappa_{c d}\left(\mathbf{r}, \mathbf{r}^{\prime}\right):=\sum_{E_{k}>0} U_{c k}^{*}(\mathbf{r}) V_{d k}\left(\mathbf{r}^{\prime}\right) .
$$


The integral operator $\hat{\Delta}$ acts on the wave function $V_{k}(\mathbf{r})$ :

$$
\left(\hat{\Delta} V_{k}\right)(\mathbf{r})=\sum_{b} \int d^{3} r^{\prime} \Delta_{a b}\left(\mathbf{r}, \mathbf{r}^{\prime}\right) V_{b k}\left(\mathbf{r}^{\prime}\right)
$$

In Ref. [11] it has been argued that in principle one should use a one-meson exchange interaction $V_{a b c d}$ in the pairing channel, as it is derived by the elimination of the mesonic degrees of freedom in the Lagrangian (1). However, it was also shown that the standard parameter sets of the mean-field approximation lead to completely unrealistic pairing matrix elements. These parameters do not reproduce the scattering data. Since at present there exists no microscopic derivation of an appropriate pairing interaction, we follow the prescription of Ref. [6], and use a phenomenological Gogny-type finite range interaction in the $p p$-channel (i.e. in Eq. 8), a procedure which requires no cut-off and which provides a very reliable description of pairing properties in finite nuclei.

$$
\begin{array}{r}
V^{p p}(1,2)=\sum_{i=1,2} e^{-\left(\left(\mathbf{r}_{1}-\mathbf{r}_{2}\right) / \mu_{i}\right)^{2}}\left(W_{i}+B_{i} P^{\sigma}\right. \\
\left.-H_{i} P^{\tau}-M_{i} P^{\sigma} P^{\tau}\right),
\end{array}
$$

with the parameters $\mu_{i}, W_{i}, B_{i}, H_{i}$ and $M_{i}(i=1,2)$.

In what follows we present results of calculation for the even-even $\mathrm{Ne}$ and $\mathrm{C}$ isotopes. For the mean-field Lagrangian the NL3 parametrization has been used. This new parameter set has been derived recently $[12$ by fitting ground state properties of a large number of spherical nuclei. Properties calculated with the NL3 effective interaction are found to be in very good agreement with experimental data for nuclei at and away from the line of beta-stability. The parameter set D1S [13] has been used for the finite range Gogny type interaction.

In Fig. 1 the rms radii for $\mathrm{Ne}(\mathrm{a})$ and $\mathrm{C}(\mathrm{b})$ isotopes are plotted as functions of neutron number. We display neutron, proton and matter $r m s$ radii, and the $\mathrm{N}^{1 / 3}$ curves. These two curves are normalized so that they coincide with neutron $r m s$ radii for ${ }^{20} \mathrm{Ne}$ and ${ }^{12} \mathrm{C}$, respectively. Neutron radii of Ne isotopes follow the $\mathrm{N}^{1 / 3}$ curve up to $\mathrm{N} \approx 22$. For larger values of $\mathrm{N}$ a sharp increase of neutron radii is observed. The matter radii follow this boost, that is, by increasing the number of neutrons, the spatial extension of the nucleus is substantially enlarged. In comparison, proton rms radii are almost constant, and only display a slow constant increase. The last bound isotope is ${ }^{40} \mathrm{Ne}$. For $\mathrm{N} \geq 32$ the neutron Fermi level becomes positive. The sudden increase in neutron rms radii, which is an indication for the halo phenomenon, is not observed for the $\mathrm{C}$ isotopes. Both the neutron and matter radii display a monotonous increase with the neutron number. The large neutron skin is a common feature in neutron-rich $\beta$-unstable nuclei. The last bound isotope is ${ }^{24} \mathrm{C}$, i.e. we find bound systems only in the s-d shell. For $\mathrm{N}>20$, in the region where for Ne the formation of the neutron halo is observed, the $\mathrm{C}$ isotopes are not bound. 
For heavier Ne isotopes $(\mathrm{N} \geq 20)$, in Fig. 2 we plot the proton and neutron density distributions. The proton density profiles do not change with the number of neutrons. The neutron density distributions display an abrupt change between ${ }^{30} \mathrm{Ne}$ and ${ }^{32} \mathrm{Ne}$. A long tail emerges, revealing the formation of a multi-particle halo. It should be noted, however, that the present calculations have been performed with the assumption of spherical symmetry, while several Ne isotopes are expected to be deformed. Therefore, what we observe is rather the trend of variations of $\mathrm{rms}$ radii with addition of pairs of neutrons. An RHB code with which deformed nuclei could be calculated, is not yet available. Nevertheless, we do not expect that the inclusion of deformation would alter the observed trend, especially for heavier Ne isotopes.

In order to understand better the formation of neutron halo in the Ne isotopes, in Fig.3 we display the neutron single-particle spectrum in the canonical basis. The energies of levels in the continuum decrease with increasing neutron number. The shell structure dramatically changes at the neutron drip $\mathrm{N} \geq 22$. The triplet of states $1 \mathrm{f}_{7 / 2}, 2 \mathrm{p}_{3 / 2}$ and $2 \mathrm{p}_{1 / 2}$ approaches zero energy, and a gap is formed between these states and all other states in the continuum. The Fermi level uniformly increases toward zero for $\mathrm{N} \leq 22$. Between $\mathrm{N}=22$ and $\mathrm{N}=32$, the Fermi level is practically constant and very close to the continuum. The addition of neutrons in this region of the drip does not increase the binding. Only the spatial extension of neutron distribution displays an increase. At $\mathrm{N}=32$ the Fermi energy becomes slightly positive, and heavier isotopes are not bound any more. The formation of the neutron halo is closely related to the quasi-degeneracy of the triplet of states $1 \mathrm{f}_{7 / 2}, 2 \mathrm{p}_{3 / 2}$ and $2 \mathrm{p}_{1 / 2}$. The role of the $1 \mathrm{f}_{7 / 2}$ is very interesting. The finite range pairing interaction promotes neutrons from the $1 \mathrm{f}_{7 / 2}$ orbital to the $2 \mathrm{p}$ levels. Since these levels are so close in energy, the total binding energy does not change significantly. Due to their small centrifugal barrier, the $2 \mathrm{p}_{3 / 2}$ and $2 \mathrm{p}_{1 / 2}$ orbitals form the halo. This is illustrated in Table 1 where we display the occupation probabilities for the triplet of states.

The details are also shown in Fig. 4a, where only the energies of these three levels and the Fermi energy are plotted as functions of the neutron number. The corresponding diagram for the $C$ isotopes is displayed in Fig. 4b. Here the energy spacings between the triplet of states are much larger, and the levels are higher in the continuum. In particular, the $1 \mathrm{f}_{7 / 2}$ level is much higher in $C$ nuclei. For $\mathrm{N}=20$, with the s-d shell fully occupied, all three levels are still high in the continuum. The pairing interaction does not have enough strength to promote particles in these levels, and as a result, heavier isotopes are unbound. Therefore, an interesting result of our calculation is that the RHB model, with finite range pairing interaction, predicts the formation of multi-neutron halo in $\mathrm{Ne}$ isotopes with two protons above the $\mathrm{Z}=8$ shell closure, but no halo is found for $C$ isotopes with two protons below the closed shell.

To illustrate the importance of the pairing interaction in the formation of the neutron halo, in Fig. 5 we display the radial dependence of the self consistent 
pairing field $\Delta(\mathrm{r})$ for three Ne nuclei. For $\mathrm{N} \leq 20$, the contribution of the pairing interaction is relatively small. We find a sharp increase in pairing between ${ }^{30} \mathrm{Ne}$ and ${ }^{32} \mathrm{Ne}$. The pairing field is concentrated on the surface of the nucleus, the matter radius increases and we observe the formation of the neutron halo. Of course the pairing field increases further with the number of neutrons, but on a much smaller scale.

In conclusion, we report results of the first application of the relativistic Hartree Bogoliubov model with finite range pairing interaction in coordinate space. In particular, we have investigated the formation of neutron halo in dripline Ne and $\mathrm{C}$ nuclei. The pairing interaction has a unique role in dripline nuclei, due to the scattering of neutron pairs from bound to continuum states. It is therefore of considerable interest to study the predictions of a finite range pairing force. We have found evidence for the formation of multi-particle neutron halo in heavier Ne isotopes, but not in $\mathrm{C}$ nuclei. Our calculations show that, in these relatively light nuclei, the halo phenomenon is caused by a delicate interplay of the self-consistent mean field, which includes all the long range $p h$ correlations, and the pairing field, which sums up the $p p$ correlations and describes the coupling to the continuum. In Ne, the triplet of almost degenerate states $1 \mathrm{f}_{7 / 2}, 2 \mathrm{p}_{3 / 2}$ and $2 \mathrm{p}_{1 / 2}$, approaches zero energy at $\mathrm{N}=20$. The pairing interaction promotes neutrons from the $1 \mathrm{f}$ to the $2 \mathrm{p}$ orbitals, and a halo is formed. In $\mathrm{C}$ on the other hand, the triplet of states is still high in the continuum at the neutron dripline, and the pairing force does not have enough strength to form a halo. The model describes the halo as a purely microscopic phenomenon. We have also performed calculations for $\mathrm{O}$ isotopes, and the results are similar to those for $\mathrm{C}$. At $\mathrm{N}=20$ the $1 \mathrm{f}_{7 / 2}$ level is still at $\approx 4 \mathrm{MeV}$ in the continuum, and ${ }^{28} \mathrm{O}$ is the last bound isotope for the NL3 parameter set. The results for N, O, F, Na and Mg will be included in a forthcoming study on the influence of the proton number on the formation of the neutron halo in the mass region above the s-d shell.

The authors thank D. Habs and G. Münzenberg for stimulating discussions. This work has been supported by the Bundesministerium für Bildung und Forschung under project 06 TM 875. D. Vretenar is Alexander von Humboldt Fellow, on leave of absence from University of Zagreb, Croatia.

\section{References}

[1] I. Tanihata, et al., Phys. Rev. Lett. 55, 2676 (1985); Phys. Lett. B206, 592 (1988).

[2] I. Tanihata, Prog. Part. Nucl. Phys. 35, 505 (1995).

[3] P. Hansen, A.S. Jensen, and B. Jonson, Annu. Rev. Nucl. Part. Phys. 45, 591 (1995). 
[4] P. Ring; Progr. Part. Nucl. Phys. 37, 193 (1996).

[5] J. Dobaczewski, H. Flocard, and J. Treiner, Nucl. Phys. A 422, 103 (1984).

[6] T. Gonzalez-Llarena, J.L. Egido, G.A. Lalazissis, and P. Ring, Phys. Lett. B 379, 13 (1996).

[7] J. Meng and P. Ring; Phys. Rev. Lett. 77 (1996) 3963.

[8] W. Pöschl, D. Vretenar, A. Rummel and P. Ring, Comput. Phys. Commun. 101, 75-107 (1997).

[9] W. Pöschl, D. Vretenar and P. Ring, Comput. Phys. Commun. (1997) in press.

[10] B.D. Serot; Rep. Prog. Phys. 55, 1855 (1992).

[11] H. Kucharek and P. Ring, Z. Phys. A 339, 23 (1991).

[12] G. A. Lalazissis, J. König and P. Ring; Phys. Rev. C55, 540 (1997).

[13] J. F. Berger, M. Girod and D. Gogny; Nucl. Phys. A428, 32 (1984). 
Table 1: 1f-2p occupation probabilities for the $N e$ isotopes.

\begin{tabular}{llll}
$\mathrm{N}$ & $1 \mathrm{f}_{7 / 2}$ & $1 \mathrm{p}_{3 / 2}$ & $1 \mathrm{p}_{1 / 2}$ \\
\hline 22 & 0.174 & 0.130 & 0.021 \\
24 & 0.291 & 0.350 & 0.064 \\
26 & 0.388 & 0.577 & 0.167 \\
28 & 0.480 & 0.749 & 0.411 \\
30 & 0.580 & 0.852 & 0.741 \\
32 & 0.709 & 0.929 & 0.924
\end{tabular}

\section{Figure Captions}

- Fig.1 Calculated rms radii for Ne (a), and C (b) isotopes as functions of neutron number.

- Fig.2 Proton and neutron density distribution for Ne isotopes.

- Fig.3 Canonical basis single-particle neutron levels as functions of the number of neutrons. The spectrum is calculated for Ne isotopes.

- Fig.4 1f-2p single-particle neutron levels in the canonical basis, for the Ne (a), and $\mathrm{C}(\mathrm{b})$ isotopes.

- Fig.5 Self consistent pairing field for ${ }^{30,32,40} \mathrm{Ne}$ 

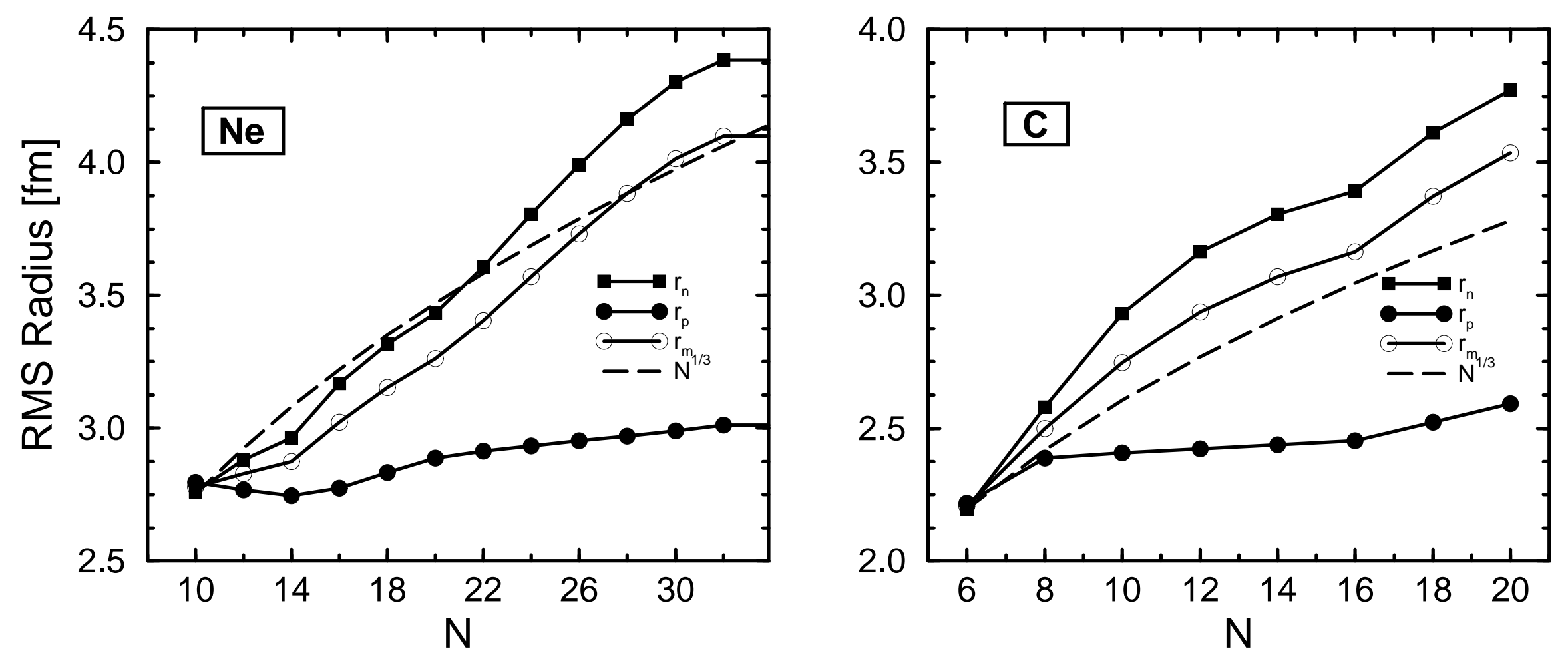


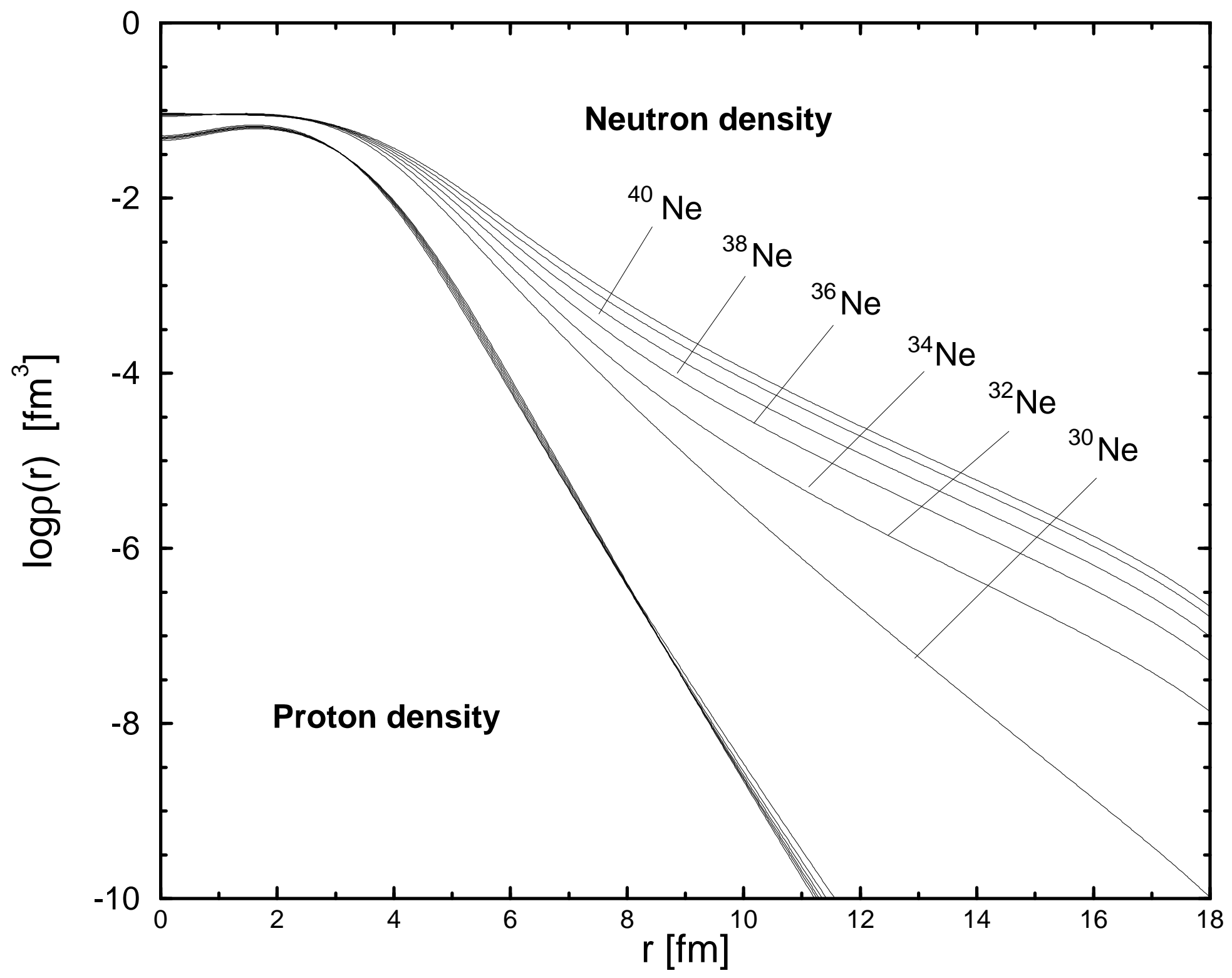




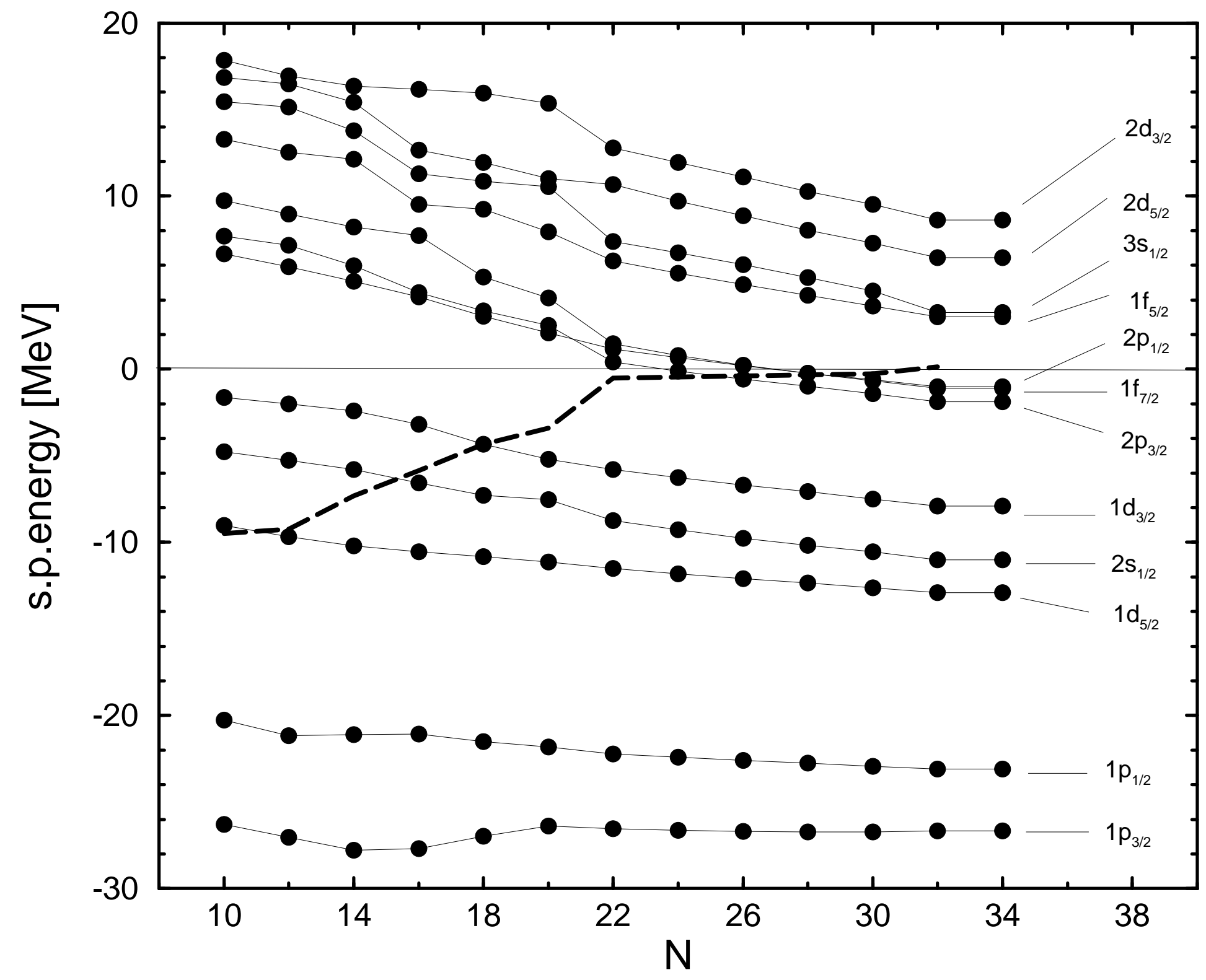




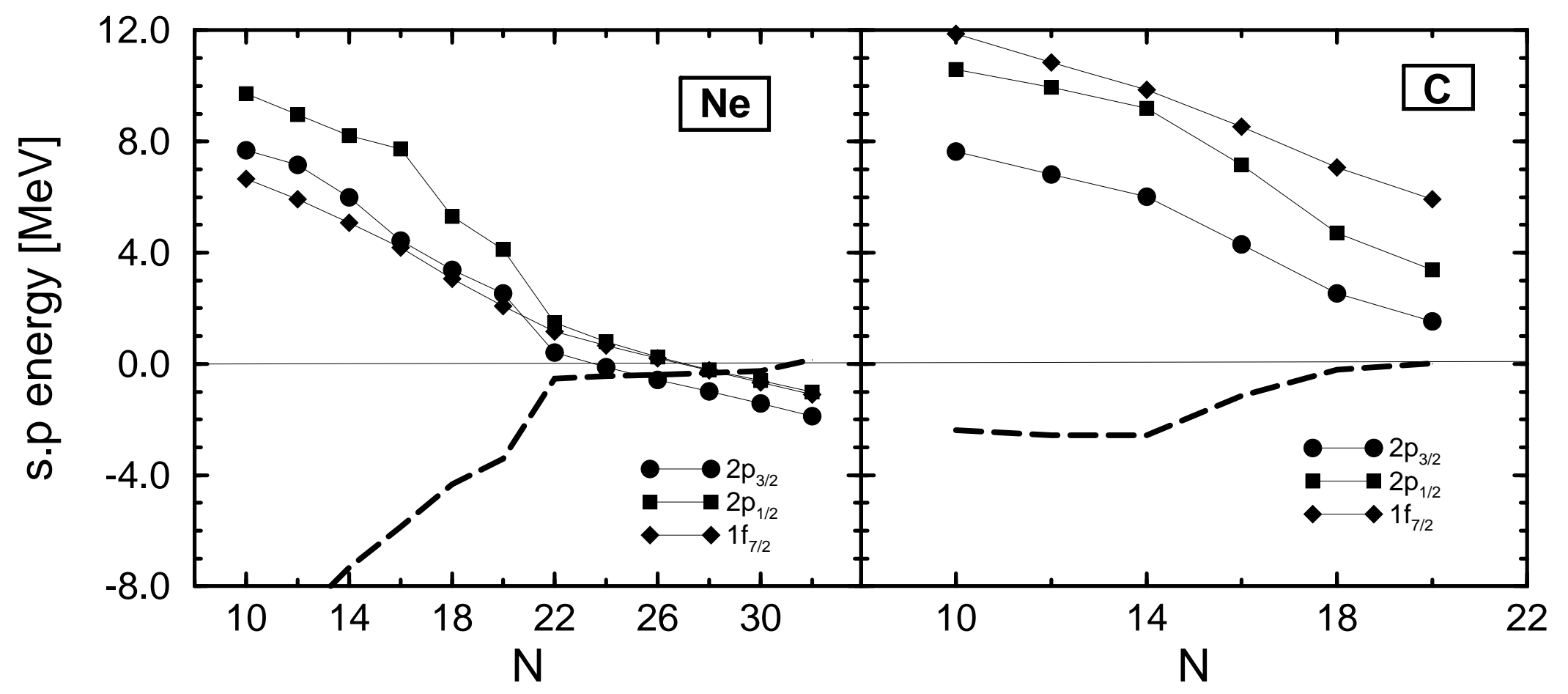




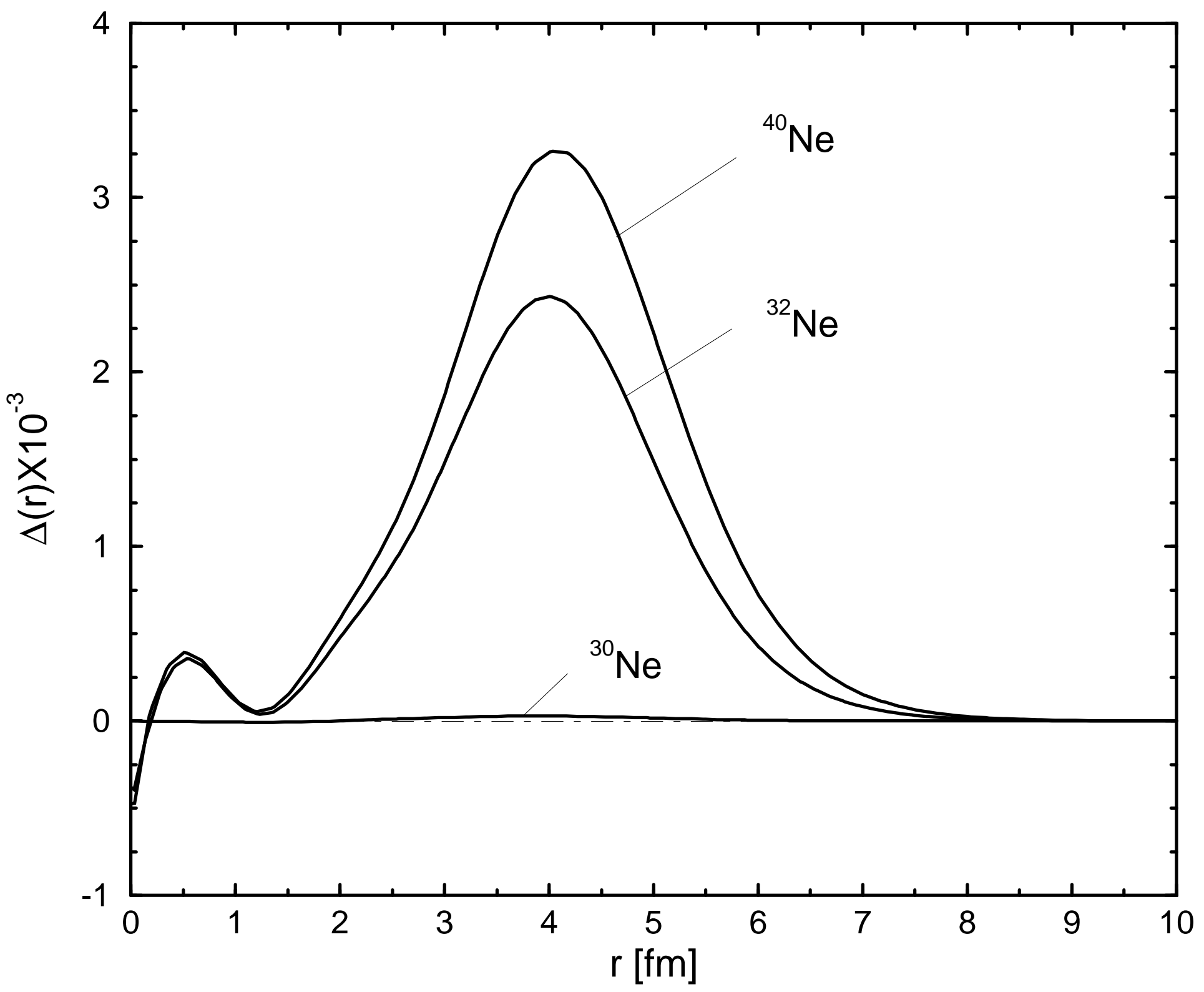

